\title{
Key factors associated with malaria infection among patients seeking care through the public sector in endemic townships of Ayeyarwady Region, Myanmar
}

Jillian Dunning ( $\sim$ jillian.dunning@gmail.com )

Clinton Health Access Initiative https://orcid.org/0000-0002-1488-0390

Nang Khaing Zar Aung

Clinton Health Access Initiative

Abigail Ward

Clinton Health Access Initiative

Moe Moe Aye

Clinton Health Access Initiative

Christopher Lourenço

Clinton Health Access Initiative

\section{Sarah Gallalee}

Clinton Health Access Initiative

\section{Stephen Lavenberg}

Clinton Health Access Initiative

Arnaud Le Menach

Clinton Health Access Initiative

\section{Myat Min Tun}

Ministry of Health: Myanmar Ministry of Health and Sports

Aung Thi

Ministry of Health: Myanmar Ministry of Health and Sports

\section{Research}

Keywords: Myanmar, Malaria, Ayeyarwady, LLIN, Rapid Diagnostic Test, Forest Transmission, Community Health Worker, Malaria Elimination

Posted Date: August 2nd, 2021

DOI: https://doi.org/10.21203/rs.3.rs-764317/v1 
License: (c) (i) This work is licensed under a Creative Commons Attribution 4.0 International License. Read Full License

Version of Record: A version of this preprint was published at Malaria Journal on March 15th, 2022. See the published version at https://doi.org/10.1186/s12936-022-04088-8. 
Key factors associated with malaria infection among patients seeking care through the public sector in endemic townships of Ayeyarwady Region, Myanmar

\section{Authors:}

Jillian Dunning ${ }^{1 *}$

Nang Khaing Zar Aung ${ }^{1}$

Abigail Ward ${ }^{1}$

Moe Moe Aye ${ }^{1}$

Christopher Lourenço ${ }^{1}$

Sarah Gallalee ${ }^{1}$

Stephen Lavenberg ${ }^{1}$

Arnaud Le Menach ${ }^{1}$

Myat Min Tun ${ }^{2}$

Aung Thi

${ }^{1}$ Clinton Health Access Initiative, Inc., Yangon, Myanmar and Boston, MA

${ }^{2}$ Myanmar Vector Borne Disease Control Program, Ministry of Health and Sports, Nay Pyi Taw, Myanmar

*Corresponding author 


\title{
Keywords
}

Myanmar; Malaria; Ayeyarwady; LLIN; Rapid Diagnostic Test; Forest Transmission; Community Health Worker; Malaria Elimination

\begin{abstract}
Background

Ayeyarwady Region in Myanmar has made significant progress towards malaria elimination, with confirmed cases decreasing from 13,522 in 2013 to 122 in 2019. As transmission declines, malaria becomes increasingly focalized both in geographic hotspots and among population groups sharing certain risk factors. Developing a thorough profile of high-risk activities associated with malaria infections is critical to ensure intervention approaches are evidence-based.
\end{abstract}

\section{Methods}

A test-negative study was conducted from September 2017 to May 2018 in Ngaputaw, Pathein and Thabaung townships in Ayeyarwady Region. Patients that presented to selected public facilities or community health volunteers with fever answered survey questions on demographic and behavioral risk factors, including exposure to malaria interventions, and were assigned to case and control groups based on the result of a malaria rapid diagnostic test. A random-effects logistic regression model adjusted for clustering at the facility level, as well as any variables along the causal pathway described by a directed acyclic graph, was used to determine odds ratios and association with malaria infections.

\section{Results}

A total of 119 cases and 1,744 controls were recruited from 41 public facilities, with a mean age of 31.3 and $63.7 \%$ percent male. Higher risk groups were identified as males (aOR 1.8, 95\% Cl: 1.2-2.9) and those with a worksite located within the forest (aOR 2.8, 95\% Cl: 1.45.3 ), specifically working in the logging (aOR $2.7,95 \% \mathrm{Cl}: 1.5-4.6$ ) and rubber plantation (aOR 3.0, 95\% Cl: 1.4-6.8) industries. Additionally, links between forest travel and malaria were observed, with risk factors identified to be sleeping in the forest within the past month (aOR 2.6, 95\% Cl: 1.1-6.3), and extended forest travel with durations from 3 to 14 days (aOR 8.6, $95 \% \mathrm{Cl}: 3.5-21.4$ ) or longer periods (aOR 8.4, 95\% Cl: 3.2-21.6).

\section{Conclusion}

Malaria transmission is highly focalized in Ayeyarwady, and results illustrate the need to target interventions to the most at-risk populations of working males and forest goers. It will become increasingly necessary to ensure full intervention coverage of at-risk populations active in forested areas as Myanmar moves closer to malaria elimination goals. 


\section{Background}

Myanmar has achieved a substantial reduction in its national malaria burden, with a $69 \%$ decline between 2015 (182,616 malaria cases [1]) and 2019 (56,411 malaria cases [2]) and achieved notable progress towards malaria elimination goals. Ayeyarwady, a delta region in the southwest of Myanmar, is indicative of this exponential decline at a regional scale. In 2015 the region accounted for $6.74 \%$ of national malaria burden with 12,312 confirmed cases [3], and in 2019 Ayeyarwady had decreased to a total of 122 (0.2\% of annual cases in Myanmar) [4]. Within this timeframe, investments in increased deployment of integrated community malaria volunteers (ICMVs) in high-risk areas, coverage of vector control interventions, and the implementation of surveillance and case management guidelines have actively contributed to this significant case decline $[5,6,7]$.

Cases are becoming increasingly focalized in certain geographies and population groups. In 2016, $96 \%$ of townships confirmed malaria cases in Ayeyarwady; in 2018, $73 \%$ of townships had reported at least one case $[8,9]$. Though malaria cases have decreased across the region, the three highest burden townships of Ngaputaw, Pathein and Thabaung represented $64 \%$ of the total malaria burden in Ayeyarwady in 2018 [6]. Incidence data from 2018 show the highest malaria burden in these three townships, located on the western coast of the region, at a rate of 0.4 cases per 1,000 population, four times the incidence rate of the regional total [6]. These contiguous townships share similar topography in levels of vegetation and forest cover, and reported a positivity rate of $18 \%$ in 2016 , with $61 \%$ of cases identified at public health facilities and hospitals and $31 \%$ identified by integrated community malaria volunteers (ICMVs), with the remaining $8 \%$ diagnosed at private facilities [10].

To catalyze malaria elimination at the regional level, outreach to remaining hotspots is necessary to interrupt onward transmission. Refined at-risk population targets can ensure prioritization of intervention coverage and developing a thorough profile of high-risk activities associated with malaria infection in these areas is critical to ensure intervention approaches are evidence-based and cost-effective. Available surveillance data do not allow for the identification of specific demographic and behavioral risk factors, and prior attempts to characterize these groups through patient exit interviews and mobile migrant population questionnaires were limited in scale [11,12]. Additional evidence is required to understand the risk factors associated with forest-related transmission.

This study reports on identified risk factors among patients seeking care from public health facilities and ICMVs in the three highest burden townships of Ayeyarwady region. Identification of risk factors and effectiveness of interventions are provided, and characteristics that define population groups as higher risk may be used to target vector control and case management interventions. 


\section{Methods}

\section{Study Population}

Study participants included any patient over two years of age, presenting to one of 17 selected health facilities or 24 integrated community malaria volunteers (ICMVs) from September 1, 2017 through May 31, 2018, who received a malaria test in accordance with national diagnosis guidelines, presenting with a fever over $38^{\circ} \mathrm{C}$, and who provided informed consent. Cases were defined as participants diagnosed with malaria confirmed by an SD BIOLINE Malaria Ag P.f/P.v rapid diagnostic test (RDT), and controls were participants with a negative RDT result.

\section{Study Design}

A test-negative study design was chosen to estimate malaria risk factors while maintaining key similarities in the control group including participation rates, diagnostic procedures, and information quality and completeness [13]. The townships of Ngaputaw, Pathein and Thabaung were selected for the study as they represent some of the highest test positivity rates within Ayeyarwady Region and also share the highest forest cover within the larger Pathein district, where natural forest covered over $37 \%$ of its land area in 2010 [14]. With forest cover greatly influencing the transmission dynamics of malaria [15], the linkages between forest cover and malaria positivity rates in Ayeyarwady persist amid increasingly focalized malaria cases. As reported malaria cases sharply declined from 2016 to 2017, study sites were selected to prioritize recruitment of positive cases. The highest burden health facilities which had the operational capacity to administer the questionnaire in addition to providing routine services were selected, determined from total reported cases from January 2016 to May 2017, which ranged from 9 to 793 [16]. Additionally, the eight highest burden villages with ICMVs were selected from each township.

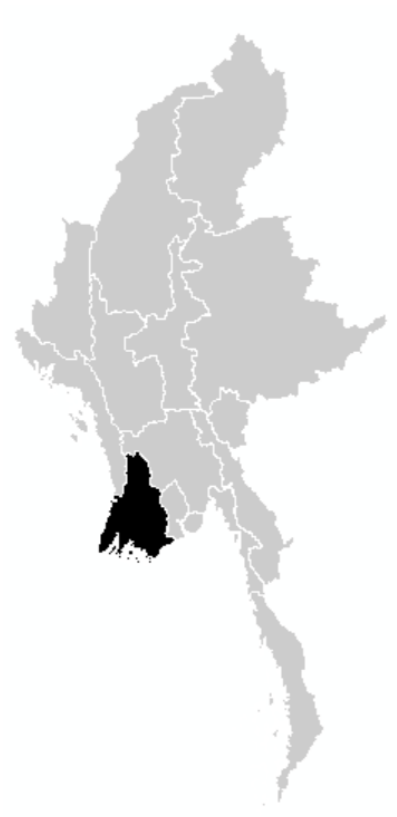

Figure 1. Ayeyarwady Region, Myanmar

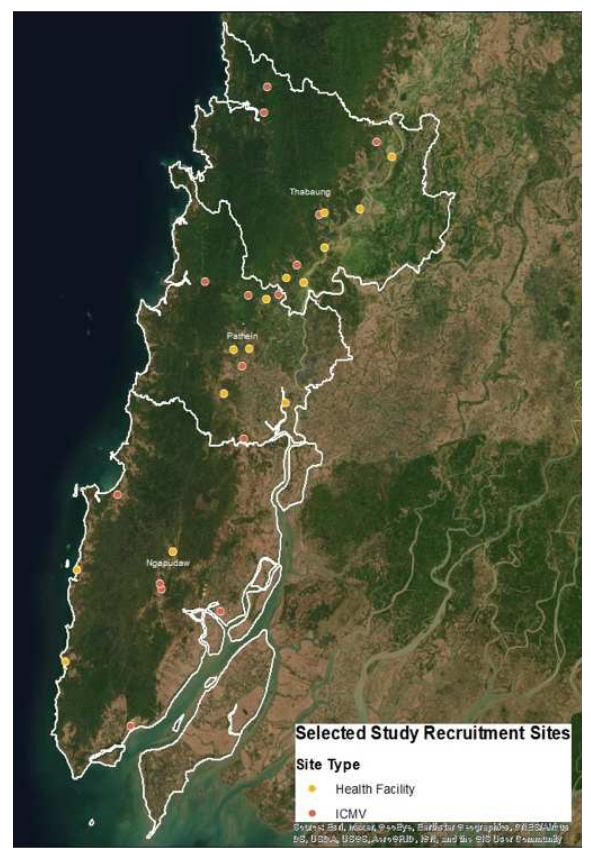

Figure 2. Selected Study Sites, HF and ICMV 
In order to detect an odds ratio of 2, assuming an alpha risk of 5\%, and a design effect of 1.5 to account for clustering at recruitment sites, a total of 625 study participants would be needed to achieve $80 \%$ power, with a case to control ratio of 1:4 [17]. To account for patient refusal, the targeted sample size was estimated at 132 cases and 525 controls. Study recruitment was timed to align with the highest transmission season in order to capture the most cases possible.

\section{Data Collection}

The survey was administered to cases and controls by basic health staff (BHS) at health facilities or by ICMVs at the point of care. Consent procedures were administered to all study participants to provide information on the study purpose and procedures, and participants were asked to provide written consent by signature. For participants younger than 18 years of age, written consent was provided by a parent or legal guardian. Written consent forms and surveys were administered by study coordinators in Burmese language. All BHS and ICMVs at selected study sites were trained on the study protocol, research ethics and informed consent, and the survey questionnaire prior to the enrollment of participants.

Individual data were collected including demographics (age, gender, occupation, education level, village of residence), whether village of residence is located in a forested area, occupation and worksite location (within or outside village), forest going behavior (frequency and duration of travel, sleeping in the forest within the past month), habitual sleeping locations, preventive methods used including bed net type, and personal history and/or contacts with a history of malaria infection in the past year. Information captured on individual history of malaria infection as well as contacts within social and professional networks was reported by study participants, and this information was not cross-referenced against patient records at health facilities.

\section{Data Processing}

Responses were recorded on paper survey forms by BHS and ICMVs and completed forms were collected on a routine basis by study coordinators. Data were entered from paper forms into Microsoft Excel and study coordinators verified concordance between electronic and paper data. Data management, cleaning, and analysis were conducted in STATA 14.2 (StataCorp, College Station, TX).

\section{Data Analysis}

Potential risk factors were assessed by logistic regression, with malaria infection as the outcome to give odds ratios and $95 \%$ confidence intervals $(\mathrm{Cl})$ for each variable. A directed acyclic graph (DAG) was developed to assess the suitability of covariates for inclusion in the final multivariable models in order to minimize the magnitude of bias for covariate estimates on the risk of malaria infection [18]. Each potential risk factor for malaria was assessed in a model including adjustment for potential confounders within the causal pathway. A binomial random-effects logit model was used for the adjusted models, with all chosen variables included as fixed effects and recruitment facility included as a random effect. A sub-group analysis of responses among participants who cited any forest travel was 
conducted with a binomial random-effects logit model on variables of interest related to forest travel and behaviors, as well as key occupation categories.

As many participants cited occupations across multiple industries, questionnaire responses were categorized into key occupations of interest in the adjusted models. Responses that included any work in the logging, rubber plantation, and farming industries were compared against all other reported occupations grouped together, as these industries represent outdoor work with remote locations and can include transitory workforces [19]. Missingness was assessed by question and the variable was removed from the adjusted model if missing data exceeded $10 \%$ of responses in case or control groups. For variables included in an adjusted model, missing data was included as a factor level in the model as its own response category.

\section{Results}

Between September 2017 and May 2018, 122 RDT positive malaria cases were enrolled in the study, along with 1,837 RDT negative controls. Thirty participants including three identified as cases were excluded from analysis due to missing informed consent forms, 36 participants were excluded as they did not meet the age requirement (at least 2 years old), and 30 participants were excluded because RDT outcome information was missing in the completed survey form and could not be assigned to case or control groups. The final sample size was 119 cases and 1,744 controls. The total cases recruited represent a sizeable portion (25\%) of malaria cases identified in the public sector of the study townships throughout this period, providing key information on the increasingly focalized population at-risk. The majority of study participants $(60.3 \%, 95 \% \mathrm{Cl}: 58.1-62.5)$ were recruited at the village level by ICMVs. Among RDT positive participants, 63.0\% (95\% Cl: 53.9-71.3) were diagnosed with Plasmodium falciparum infection, 33.6\% (95\% Cl: $25.6-42.6)$ with $P$. vivax, and $3.4 \%(95 \% \mathrm{Cl}: 1.2-8.7)$ with mixed infection. Mean age among cases was 26.7 years (95\% Cl: 24.0-29.4), slightly younger than control participants with a mean of 31.6 years (95\% Cl: 30.9-32.4), though there were no significant differences in age groups between cases and controls. Occupations that included more than one industry were reported in $3.6 \%$ (95\% Cl: $2.8-4.6)$ of participants, with $1.5 \%$ (95\% Cl: $1.0-2.1)$ citing work in two or more of the logging, rubber plantation, and farming industries (Table 1). Of participants who reported logging work as an occupation, 97.8\% (95\% Cl: 95.7-98.9) reported worksites located in forested areas and $78.0 \%(95 \% \mathrm{Cl}: 73.5-81.9)$ had slept in the forest within the past month.

In bivariate analysis, confirmed malaria cases were more likely to be male (OR $1.7,95 \% \mathrm{Cl}$ : 1.1-2.8), work in logging (OR 2.6, 95\% Cl: 1.7-4.2) or rubber plantations (OR 3.4, 95\% Cl: 1.8-6.8), have a worksite located within the forest (OR 3.0, 95\% Cl: 1.9-4.7), stay within the forest for a period longer than 3 days, and report that they had malaria within the past year (OR 9.5, 95\% Cl 5.8-15.4). To examine the potential impact of relapse on participant responses of past malaria among cases confirmed with P.vivax infection, a chi-square test of independence was used to determine any association between malaria infection species and self-reported malaria within the past year and did not find a statistically significant association. Cases were less likely to use a bed net in the home (OR 0.6, 95\% $\mathrm{Cl}: 0.4-0.9$ ) or 
an LLIN/ITN in the forest (OR $0.5,95 \% \mathrm{Cl}: 0.3-0.9)$, and refraining from forest travel showed a protective effect (OR $0.2,95 \% \mathrm{Cl}: 0.1-0.5)$. Table 1 shows the demographics of study participants included in the sample and bivariate odds ratios.

Table 1. Demographic and behavioral characteristics of 1,863 study participants, bivariate analysis odds ratio (OR) and 95\% confidence intervals (95\% Cls) for malaria infection against each covariate.

\begin{tabular}{|c|c|c|c|}
\hline & $\begin{array}{c}\text { Cases } \\
{[n=119]} \\
\%(95 \% \mathrm{Cl})\end{array}$ & $\begin{array}{c}\text { Controls } \\
{[n=1,744]} \\
\%(95 \% \mathrm{Cl})\end{array}$ & $\begin{array}{l}\text { Unadjusted OR } \\
\quad(95 \% \mathrm{Cl})\end{array}$ \\
\hline \multicolumn{4}{|l|}{ Age Category } \\
\hline Age 2-14 & $14.3(9.0-21.9)$ & $15.5(13.9-17.3)$ & $0.9(0.5-1.5)$ \\
\hline Age 15- 59* & $84.0(76.2$ - 89.6) & $79.5(77.5-81.3)$ & 1 \\
\hline Age 60 - 90 & $1.7(0.4-6.6)$ & $4.9(4.0-6.1)$ & $0.3(0.1-1.3)$ \\
\hline \multicolumn{4}{|l|}{ Sex } \\
\hline Male & $73.9(65.2$ - 81.1) & $63.1(60.8-65.3)$ & $1.7(1.1-2.8)$ \\
\hline Female* & $22.7(15.9-31.2)$ & $33.2(31.0-35.4)$ & 1 \\
\hline Missing & $3.4(1.2-8.7)$ & $3.7(2.9-4.7)$ & $1.3(0.4-3.9)$ \\
\hline \multicolumn{4}{|l|}{ Township } \\
\hline Ngaputaw* & $45.4(36.6-54.5)$ & $27.5(25.5-29.7)$ & 1 \\
\hline Pathein & $36.9(28.7-46.1)$ & $43.6(41.3-45.9)$ & $0.5(0.3-0.8)$ \\
\hline Thabaung & $17.6(11.7-25.6)$ & $28.8(26.8-31.0)$ & $0.4(0.2-0.6)$ \\
\hline \multicolumn{4}{|l|}{ Education } \\
\hline Illiterate/Never Attended School* & $4.2(1.7-9.8)$ & $4.8(3.9-5.9)$ & 1 \\
\hline Able to Read and Write & $9.2(5.2-16.0)$ & $20.2(18.4-22.1)$ & $0.5(0.2-1.5)$ \\
\hline Primary School & $52.1(43.1-61.0)$ & $44.8(42.4-47.1)$ & $1.3(0.5-3.4)$ \\
\hline Middle School & $31.1(23.4-40.0)$ & $21.5(19.7-23.5)$ & $1.6(0.6-4.3)$ \\
\hline High School & $2.5(0.8-7.6)$ & $6.8(5.7-8.0)$ & $0.4(0.1-1.8)$ \\
\hline University Student/Graduate & $0.8(0.1-5.8)$ & $1.1(0.74-1.8)$ & $0.8(0.1-7.6)$ \\
\hline Missing & 0 & $0.7(0.4-1.2)$ & - \\
\hline \multicolumn{4}{|l|}{ Occupation } \\
\hline Any logging work & $33.6(25.6-42.7)$ & $19.1(17.3-21.0)$ & $2.6(1.7-4.2)$ \\
\hline Any farming work & $20.2(13.8-28.4)$ & $24.1(22.1-26.1)$ & $1.2(0.7-2.1)$ \\
\hline Any rubber plantation work & $10.9(6.4-18.0)$ & $4.7(3.8-5.9)$ & $3.4(1.8-6.8)$ \\
\hline Two or more of the above & $2.5(0.8-7.6)$ & $1.4(0.9-2.1)$ & $2.6(0.8-9.1)$ \\
\hline Other occupation* & $32.8(24.8-41.8)$ & $49.2(46.8-51.5)$ & 1 \\
\hline Missing & 0 & $1.4(0.9-2.1)$ & - \\
\hline \multicolumn{4}{|l|}{ Recruitment Point Type } \\
\hline Health Facility* & $46.2(37.4-55.3)$ & $39.2(36.9-41.5)$ & 1 \\
\hline ICMV & $53.8(44.7-62.6)$ & $60.8(58.5-63.0)$ & $0.7(0.5-1.1)$ \\
\hline \multicolumn{4}{|l|}{ Village located in the forest } \\
\hline Yes & $48.7(39.8-57.8)$ & $46.8(44.4-49.1)$ & $1.1(0.7-1.6)$ \\
\hline No* & $47.0(38.2-56.2)$ & $49.0(46.7-51.4)$ & 1 \\
\hline
\end{tabular}


Worksite located in the forest (outside of home village)

\begin{tabular}{|c|c|c|c|}
\hline Yes & $76.5(67.9-83.3)$ & $51.4(49.1-53.8)$ & $3.0(1.9-4.7)$ \\
\hline No* & $22.7(15.9-31.2)$ & $46.7(44.3-49.0)$ & 1 \\
\hline Missing & $0.8(0.1-5.9)$ & $1.9(1.3-2.6)$ & $0.9(0.1-6.9)$ \\
\hline \multicolumn{4}{|l|}{ Duration of forest travel } \\
\hline No forest travel* & $10.1(5.8-17.0)$ & $35.6(33.4-37.9)$ & 1 \\
\hline Less than 3 days & $28.6(21.1$ - 37.4) & $33.1(30.9-35.4)$ & $3.0(1.6-5.9)$ \\
\hline 3-14 days & 44.5 (35.7 - 53.7) & $21.9(20.1$ - 23.9) & $7.2(3.8-13.6)$ \\
\hline Longer than 14 days & $15.1(9.7-22.9)$ & $3.4(2.7-4.4)$ & $15.5(7.1-33.8)$ \\
\hline Missing & $1.7(0.4-6.6)$ & $5.8(4.8-7.0)$ & $1.0(0.2-4.6)$ \\
\hline \multicolumn{4}{|l|}{ Slept in the Forest Within the Past Month } \\
\hline Yes & $75.6(66.9-82.6)$ & $39.0(36.8-41.4)$ & $5.0(3.2-7.9)$ \\
\hline No* & $21.0(14.5-29.4)$ & $54.6(52.3-56.9)$ & 1 \\
\hline Missing/Don't know & $3.4(1.2-8.7)$ & $6.3(5.2-7.5)$ & $1.4(0.5-4.0)$ \\
\hline \multicolumn{4}{|l|}{ Prevention methods used at home } \\
\hline Preventive methods include bed nets & $78.8(70.4-85.3)$ & $85.8(84.0-87.4)$ & $0.6(0.4-0.9)$ \\
\hline Preventive methods do not include bed nets* & $21.2(15.2-30.3)$ & $14.2(12.6-15.9)$ & 1 \\
\hline \multicolumn{4}{|l|}{ Prevention methods used in the forest } \\
\hline Preventive methods include bed nets & $53.8(13.8-28.7)$ & $46.4(44.0-48.7)$ & $2.1(1.3-3.3)$ \\
\hline Preventive methods do not include bed nets* & $20.2(13.8-28.5)$ & $35.8(33.6-38.1)$ & 1 \\
\hline No preventive methods & $26.0(18.9-34.8)$ & $17.8(16.0-19.6)$ & $2.6(1.5-4.5)$ \\
\hline \multicolumn{4}{|l|}{ Type of net used while sleeping in the forest } \\
\hline Conventional Net* & $26.9(19.6-35.7)$ & $15.5(13.9-17.3)$ & 1 \\
\hline LLIN/ITN & $21.0(14.5-29.4)$ & $22.2(20.3-24.3)$ & $0.5(0.3-0.9)$ \\
\hline Hammock Net & 0 & $0.1(0.01-0.4)$ & - \\
\hline Use another form of prevention & $5.9(2.8-11.9)$ & $4.8(3.9-5.9)$ & $0.7(0.3-1.6)$ \\
\hline Don't use any preventive methods & $22.7(15.9-31.2)$ & $12.5(11.0-14.1)$ & $1.0(0.6-1.8)$ \\
\hline No reported forest travel & $5.0(2.2-10.9)$ & $13.5(12.0-15.2)$ & $0.2(0.1-0.5)$ \\
\hline Missing/Don't know & $18.5(12.4-26.6)$ & $31.3(29.2-33.5)$ & $0.3(0.2-0.6)$ \\
\hline \multicolumn{4}{|l|}{ Self-reported malaria infection in the past year } \\
\hline \multicolumn{4}{|l|}{ Respondent infected } \\
\hline (or respondent and someone else among contacts) & $30.1(22.2$ - 39.3) & $4.5(3.6-5.6)$ & $9.5(5.8-15.4)$ \\
\hline $\begin{array}{l}\text { Friends, family, coworkers, other contact infected } \\
\qquad \text { (respondent not infected) }\end{array}$ & $15.0(9.5-23.0)$ & $17.4(15.7-19.4)$ & $1.2(0.7-2.1)$ \\
\hline No one respondent knows infected* & $52.2(42.9-61.4)$ & $73.8(71.6-75.9)$ & 1 \\
\hline Missing/Don't know & $2.6(0.8-8.0)$ & $4.2(3.3-5.3)$ & $0.8(0.3-2.9)$ \\
\hline
\end{tabular}

*Baseline category.

$\mathrm{Cl}=$ confidence interval. $a \mathrm{OO}=$ adjusted odds ratio. ICMV = integrated community malaria volunteer. $L L I N=$ Long-lasting insecticide treated net, ITN = Insecticide treated net 
The final adjusted multivariable models identified significant risk groups as males (aOR 1.8, 95\% $\mathrm{Cl} 1.2-2.9$ ) and those reporting a worksite located in the forest (aOR 2.8, 95\% $\mathrm{Cl}: 1.4-$ 5.3 ), particularly any work in the logging (aOR $2.7,95 \% \mathrm{Cl}: 1.5-4.6$ ) and rubber plantation (aOR 3.0, 95\% Cl:1.4-6.8) industries. Individuals traveling to the forest for 3 to 14 days (aOR 8.6, 95\% Cl: 3.5-21.4) and periods of 14 days or longer (aOR 8.4, 95\% Cl: 3.2-21.6) are also at a significantly higher risk (Table 2 ).

\section{Forest Going Populations}

A total of 1,224 respondents including 107 cases and 1,117 controls (65\% of total sample) reported any forest travel, with varied frequencies. Forest travel was defined as traveling through or staying in the forest outside of the home, in order to estimate the effect of longer-term forest related economic activity as well as increased mosquito exposure from sleeping outdoors or in temporary structures. Within the subset of forest goers, no significant differences were found between case and control groups in the use of preventive methods in the forest nor in the type of bed net used, after adjusting for age group, worksites located in the forest, duration of forest travel/sleeping in the forest, and point of care (Table 2). 
Table 2. Adjusted odds ratios odds ratio (aOR) and $95 \%$ confidence intervals ( $95 \% \mathrm{Cls}$ ) for malaria infection against each covariate

\begin{tabular}{|c|c|}
\hline Exposure & aOR, Cl \\
\hline \multicolumn{2}{|l|}{ Age Category ${ }^{1}$} \\
\hline Age $2-14$ & $0.8(0.4-1.4)$ \\
\hline Age 15-59* & 1 \\
\hline Age 60-90 & $0.4(0.1-1.7)$ \\
\hline \multicolumn{2}{|l|}{ Sex ${ }^{1}$} \\
\hline Male & $1.8(1.2-2.9)$ \\
\hline Female* & 1 \\
\hline Missing & $1.47(0.5-4.6)$ \\
\hline \multicolumn{2}{|l|}{ Education ${ }^{2}$} \\
\hline Illiterate/Never Attended School* & 1 \\
\hline Able to Read and Write & $0.3(0.1-1.1)$ \\
\hline Primary School & $0.9(0.3-2.5)$ \\
\hline Middle School & $1.1(0.4-2.9)$ \\
\hline High School & $0.26(0.1-1.2)$ \\
\hline University Student/Graduate & $0.4(0.1-4.9)$ \\
\hline Missing & $1^{*}$ \\
\hline \multicolumn{2}{|l|}{ Occupation ${ }^{3}$} \\
\hline Any logging work & $2.7(1.5-4.6)$ \\
\hline Any farming work & $1.1(0.6-2.0)$ \\
\hline Any rubber plantation work & $3.0(1.4-6.8)$ \\
\hline Two or more of the above & $3.3(0.8-12.8)$ \\
\hline Other occupation* & 1 \\
\hline Missing & $1^{*}$ \\
\hline \multicolumn{2}{|l|}{ Worksite located in the forest (outside of home village) ${ }^{4}$} \\
\hline Yes & $2.8(1.4-5.3)$ \\
\hline No* & 1 \\
\hline Missing & $2.7(0.3-26.4)$ \\
\hline \multicolumn{2}{|l|}{ Duration of forest travel ${ }^{5}$} \\
\hline No forest travel* & 1 \\
\hline Less than 3 days & $2.4(0.9-5.7)$ \\
\hline 3-14 days & $8.6(3.5-21.4)$ \\
\hline Longer than 14 days & $8.4(3.2-21.6)$ \\
\hline Missing & $1.5(0.3-7.3)$ \\
\hline \multicolumn{2}{|l|}{ Slept in the Forest Within the Past Month ${ }^{6}$} \\
\hline Yes & $2.6(1.1-6.3)$ \\
\hline No* & 1 \\
\hline Missing/Don't know & $1.8(0.2-12.4)$ \\
\hline \multicolumn{2}{|l|}{ Preventive methods used in the forest ${ }^{7}$} \\
\hline Preventive methods include bed nets & $0.6(0.3-1.3)$ \\
\hline
\end{tabular}




\begin{tabular}{rr}
$\begin{array}{r}\text { No preventive methods } \\
\text { Missing/Don't know }\end{array}$ & $1.6(0.7-3.7)$ \\
\hline Type of net used while sleeping in the forest ${ }^{7}$ & $2.6(0.9-7.5)$ \\
Conventional Net* & 1 \\
LLIN/ITN & $0.6(0.3-1.1)$ \\
Hammock Net & $1^{*}$ \\
Use another form of prevention & $1.4(0.7-2.7)$ \\
Don't know & $1.4(0.4-4.6)$ \\
Missing & $2.1(0.7,6.7)$ \\
\hline
\end{tabular}

* Baseline categories, $a O R=1$ * indicates too few observations

1. adjusted for point of care (random effect)

2. adjusted for age, sex, and point of care

3. adjusted for age and point of care

4. adjusted for age, occupation and point of care

5. adjusted for age, worksite in the forest, and point of care

6. adjusted for age, duration of forest travel, worksite in the forest, and point of care

7. adjusted for age, sleeping in the forest, and point of care

$\mathrm{Cl}=$ confidence interval. $a \mathrm{O}=$ adjusted odds ratio. ICMV = integrated community malaria volunteer.

$L L I N=$ Long-lasting insecticide treated net, ITN = Insecticide treated net

\section{Discussion}

Among those seeking care in the public sector in endemic townships in Ayeyarwady Region, those presenting with fever are mostly male, working in forested areas and in outdoor occupations of rubber plantations and logging, often traveling to the forest for extended periods and sleeping away from the home. Those with a history of malaria or have social and professional connections to those with a history of malaria also appear to be at higher risk, though the causal linkage to malaria infection cannot be directly established. Among forest travelers, regular use of preventive methods while in the forest and use of an insecticide treated net did not significantly reduce the risk of malaria when adjusting for the effect of age, point of care, and sleeping in the forest.

Villages of residence for cases and controls were located around the forest fringe, and the catchment areas of selected health facilities include several registered worksites in the logging and agricultural industries. When asked for village of residence, several study participants reported the location or company name of their employer, suggesting lengthy periods of time away from home to pursue economic opportunities and worksite related travel in forested areas. Increased risk for those with worksites in the forest contrasted to no significant risk identified for those living within a village in the forest suggests the characteristics of lodging and occupational behaviors and practices for forest work provide opportunity for malaria transmission. Travel in forested areas [20] and for occupational 
purposes $[21,22]$ among males $[23,24]$ have shown to be associated with malaria risk in other studies conducted in low endemic and elimination settings. Sleeping outside of the household has also been identified as a risk factor in other contexts $[25,26]$ and these results confirm the linkage to these occupational and forest travel components within the townships in Ayeyarwady, while also providing additional details on preventive method use specifically among the population of forest goers. Study results consistent with existing literature reinforce the potential impact of mechanisms such as expanding diagnosis services directly at work sites and ensuring access to prevention and treatment services among groups traveling and working in forested areas for extended periods of time.

While the study findings inform the profile of high-risk activities, a mix of intervention approaches may be needed as locally tailored strategies are necessary to interrupt malaria transmission [27]. Higher malaria risk among those citing any work in logging and rubber plantation industries suggests the malaria cases that persist in Ayeyarwady Region are perpetuated by communities connected socially and professionally, and the most effective outreach methods to target connected groups should consider approaches proven successful in this context. Case contact tracing and high-risk group screening may facilitate the identification and treatment of connected malaria cases [28]. Findings in Myanmar indicate that passive ICMV testing has higher positivity rates compared to mobile clinics [29] and that active case detection in response to positive cases does not necessarily identify additional cases in low endemic areas [30]. Expanding health services provided by ICMVs in hard-to-reach areas [31] or within the catchment of forest worksites may accelerate progress in malaria decline. An emphasis on improved case management practices to ensure high coverage of testing and treatment, as well as strong case and foci investigation and response within these connected communities, would impact the acceleration of the decline of malaria cases. These survey findings clarify risk factors and suggest further direction for additional investigation to tailor interventions appropriately. Interventions that have been implemented in limited settings or have provided less evidence of effectiveness within the context of Southeast Asia such as mass drug administration [32, 33], stand-by presumptive treatment and chemoprophylaxis [34], topical repellents [35], insecticide treated clothing [36], and insecticide treated hammock nets [37] would not be interchangeable with core interventions around surveillance and case management, and study results indicate that targeting forest workers and ensuring the acceptability of interventions among this group is a critical component to an effective malaria elimination strategy.

No associations were found between bed net usage and malaria infection at home, or among forest going populations in the multivariate analysis. A substantial proportion of case participants $(26.9 \%)$ reported use of a bed net that is not treated with insecticide in the forest though endemic areas continue to be targeted by LLIN campaigns, which prompts further questions around the distribution of LLINs among high-risk groups, as well as available options for regular re-treatment of bed nets. Information on additional malaria preventive methods (including topical and spatial mosquito repellents) in forested areas were not collected in this survey. Information around sleeping arrangements and behavior during outdoor biting hours, including the feasibility and acceptability of preventive methods within the context of forest work, would improve the identification of potential 
exposures within this population and allow better targeting of vector control interventions and more effective malaria prevention.

There were several limitations as a result of study design. Survey administration was incorporated into routine health care and consequently may underrepresent characteristics of populations who do not seek treatment at health care facilities, which may include seasonal workers and migrant populations. In order to mitigate this potential effect, eight ICMVs were selected in addition to six health facilities in each study township, under the assumption that hard to reach populations would be more likely to present to a health worker at the village level. Additionally, study participant selection was limited to those over two years of age, which led to the exclusion of 36 participants from the study and impacts generalizability to younger age groups. Survey administrators were routine health care providers who are responsible for providing bed nets and health promotion activities to the study communities. Consequently, participants may have felt the need to overstate use of malaria preventive methods and bed net usage. Finally, self-reported frequency and duration of forest travel as well as use of preventive methods could be affected by recall bias. Study recruitment was targeted towards high-burden facilities within high-burden townships, which limited the representativeness of the study population to other geographies in Myanmar.

\section{Conclusion}

This study identified several risk factors for malaria transmission that present a continuous challenge to elimination efforts. In this setting, sleeping in the forest in the past month, and a prior history of malaria infection were found to be the strongest risk factors for malaria. Ensuring that receptive areas, such as worksites where employees are sleeping in forested areas outdoors and overnight, are prioritized for intervention coverage could have an impact in the prevention of local malaria transmission. As a result of these findings, designing a package of interventions targeted to those that travel and work in and around the forest could catalyze progress toward elimination in Ayeyarwady Region. 


\section{Abbreviations and Acronyms}

aOR

BHS

CHAI

$\mathrm{Cl}$

DAG

GMS

HF

ICMV

ITN

LLIN

NMCP

$\mathrm{OR}$

RDT
Adjusted Odds Ratio

Basic Health Staff

Clinton Health Access Initiative

Confidence Interval

Directed Acyclic Graph

Greater Mekong Subregion

Health Facility

Integrated Community Malaria Volunteer

Insecticide Treated Net

Long-Lasting Insecticide Treated Net

National Malaria Control Program

Odds Ratio

Rapid Diagnostic Test 


\section{Declarations}

\section{Ethics Approval and Consent to Participate}

Institutional Review Board approval was obtained for this study from the University of Public Health (Yangon, Myanmar) and Advarra (Columbia, MD, USA), protocol reference number Pro00022967.

\section{Consent for Publication}

Not applicable.

\section{Availability of Data and Materials}

The data that support the findings of this study are not publicly available due to data protection from the Vector Borne Disease Control Program in Myanmar. Aggregate and anonymized data can be available from the corresponding author upon reasonable request and with permission of the Myanmar Vector Borne Disease Control Program, Ministry of Health and Sports.

\section{Competing Interests}

The authors declare they have no competing interests.

\section{Funding}

This work was supported by the Bill \& Melinda Gates Foundation [OPP1109772]. Under the grant conditions of the Foundation, a Creative Commons Attribution 4.0 Generic License has already been assigned to the Author Accepted Manuscript version that might arise from this submission. The funder had no role in the design or implementation of the study, interpretation of the data, or writing of the manuscript. 


\section{Acknowledgements}

MAP (Katherine Battle)

CHAI (Joseph Novotny, Julia Dunn)

Dr May Thin Ko, Team Leader (Malaria), Pathein District, Ayeyarwady region

U Win Hlaing, Malaria Assistant (retired), Vector Borne Disease Control, Ayeyarwady region

U Mg Mg Aye, Malaria Assistant, Regional Vector Borne Disease Control, Ayeyarwady region

U Than Htay, Malaria supervisor, Township Vector Borne Disease Control, Ngaputaw township, Ayeyarwady region

U Thura Shwe, Malaria supervisor, Township Vector Borne Disease Control, Pathein township, Ayeyarwady region

U Win Kyaw, Malaria supervisor, Township Vector Borne Disease Control, Thabaung township, Ayeyarwady region

Bill \& Melinda Gates Foundation

\section{Affiliations}

Clinton Health Access Initiative, Yangon, Myanmar

Jillian Dunning, Moe Moe Aye, Nang Khaing Zar Aung, Stephen Lavenberg, Sarah Gallalee

Clinton Health Access Initiative, Boston, MA, USA

Abigail Ward, Arnaud Le Menach

Clinton Health Access Initiative, Vientiane, Laos

Christopher Lourenço

Myanmar Vector Borne Disease Control Program, Ministry of Health and Sports, Nay Pyi Taw, Myanmar

Myat Min Tun, Aung Thi

\section{Contributions}

JD and CL conceptualized the study. JD, AW, MMA, and NKZA developed the study protocol, trained health facility and community health volunteer data collectors, compiled and cleaned the data, performed the analysis, interpreted results, and prepared the initial manuscript. ALM, CL, and MMT supported the interpretation of results. SG, SL, and AT contributed to the final manuscript. All authors reviewed and approved the final manuscript. 


\section{Supporting Information}

Causal directed acyclic graphs (DAG). The figure represents the causal diagram for the data.

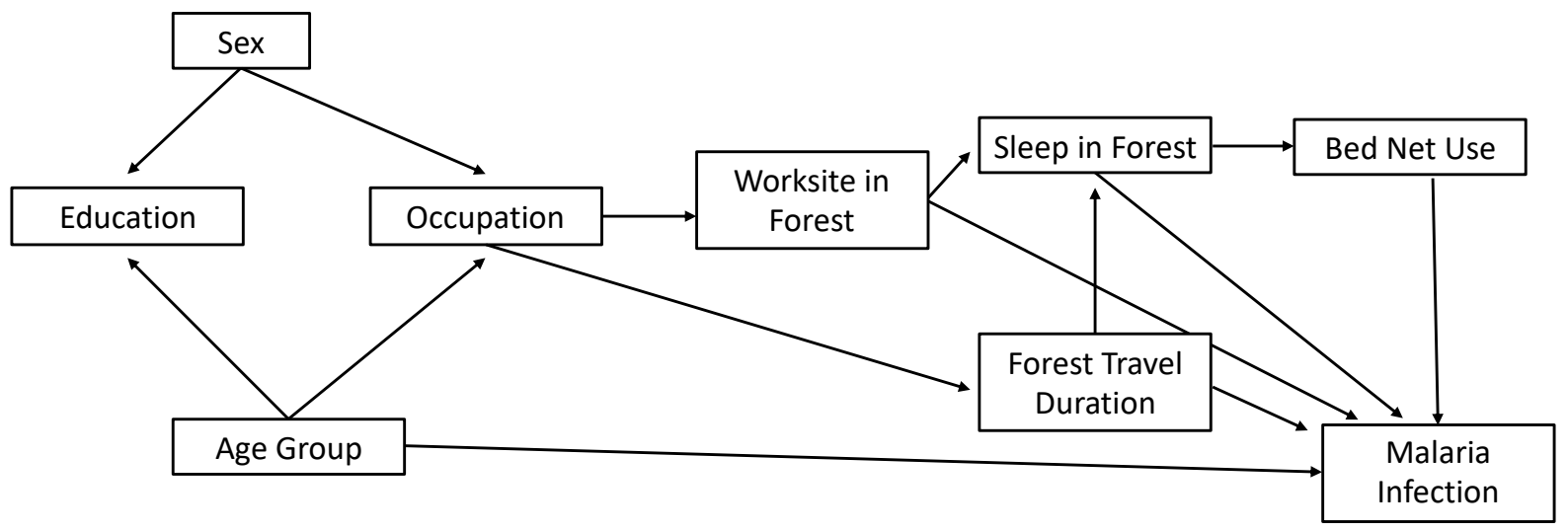




\section{References}

1. Vector Borne Disease Control Program: 2016 Annual Review Meeting. 2017.

2. World Health Organization. "Mekong Malaria Elimination, Epidemiology Summary." Volume 5, May 2020.

3. Vector Borne Disease Control Program: 2016 Annual Review Meeting. 2017.

4. Vector Borne Disease Control Program: 2019 Annual Review Meeting. 2020.

5. Vector Borne Disease Control Program: Annual Vector Borne Disease Control Report Ayeyarwady Region. Ministry of Health and Sports; 2017.

6. Graves P, Kyaw MP, Laihad F, Aung PL: Myanmar Malaria Burden Reduction Assessment. USAID, PMI; 2019.

7. Gallalee, S., Ward, A., Aye, M., Aung, N. K., Dunn, J., Lourenco, C., ... \& Tun, M. M. (2020). Factors associated with the decline of malaria in Myanmar's Ayeyarwady Region between 2013 and 2017. Manuscript in preparation.

8. Vector Borne Disease Control Program: 2016 Annual Review Meeting. 2017.

9. Vector Borne Disease Control Program: 2018 Annual Review Meeting. 2019.

10. Vector Borne Disease Control Program: 2016 Annual Review Meeting. 2017.

11. Vector Borne Disease Control Program: Mobile Migrant Population Survey, Ministry of Health and Sports; 2017.

12. Vector Borne Disease Control Program: Ayeyarwady Patient Exit Interview, Ministry of Health and Sports; 2017.

13. Vandenbroucke, Jan P.a,b,c; Pearce, Neild Test-Negative Designs, Epidemiology: November 2019 - Volume 30 - Issue 6 - $\mathrm{p}$ 838-844

14. Global Forest Watch, 2014. World Resources Institute. Accessed on Oct 2, 2019.

www.globalforestwatch.org.

15. Kar, N. P., Kumar, A., Singh, O. P., Carlton, J. M., \& Nanda, N. (2014). A review of malaria transmission dynamics in forest ecosystems. Parasites \& vectors, 7(1), 1-12.

16. Vector Borne Disease Control Program: 2017 Annual Review Meeting. 2018.

17. Eldridge SM, Ashby D, Kerry S. 2006 Sample size for cluster randomized trials: effect of coefficient of variation of cluster size and analysis method. Int J Epidemiol 35(5): 1292-300.

18. Lederer, D. J., Bell, S. C., Branson, R. D., Chalmers, J. D., Marshall, R., Maslove, D. M., ... \& Vincent, J. L. (2019). Control of confounding and reporting of results in causal inference studies. Guidance for authors from editors of respiratory, sleep, and critical care journals. Annals of the American Thoracic Society, 16(1), 22-28. 19. Population Services International, Myanmar. (2016). Malaria-at-risk Worksites Mapping Survey Tier 1 Report.

20. Herdiana, H., Cotter, C., Coutrier, F. N., Zarlinda, I., Zelman, B. W., Tirta, Y. K., ... \& Hsiang, M. S. (2016). Malaria risk factor assessment using active and passive surveillance data from Aceh Besar, Indonesia, a low endemic, malaria elimination setting with Plasmodium knowlesi, Plasmodium vivax, and Plasmodium falciparum. Malaria journal, 15(1), 468.

21. Bhumiratana, A., Sorosjinda-Nunthawarasilp, P., Kaewwaen, W., Maneekan, P., \& Pimnon, S. (2013). Malaria-associated rubber plantations in Thailand. Travel Medicine and Infectious Disease, 11(1), 37-50.

22. Guyant, Philippe, et al. "Malaria and the mobile and migrant population in Cambodia: a population movement framework to inform strategies for malaria control and elimination." Malaria journal 14.1 (2015): 252.

23. Alemu, K., Worku, A., Berhane, Y., \& Kumie, A. (2014). Men traveling away from home are more likely to bring malaria into high altitude villages, northwest Ethiopia. PLoS One, 9(4).

24. Littrell, M., Sow, G. D., Ngom, A., Ba, M., Mboup, B. M., Dieye, Y., ... \& Steketee, R. W. (2013). Case investigation and reactive case detection for malaria elimination in northern Senegal. Malaria journal, 12(1), 331.

25. Smith, J. L., Auala, J., Haindongo, E., Uusiku, P., Gosling, R., Kleinschmidt, I., ... \& Sturrock, H. J. (2017).

Malaria risk in young male travellers but local transmission persists: a case-control study in low transmission Namibia. Malaria journal, 16(1), 70.

26. Yukich, J. O., Taylor, C., Eisele, T. P., Reithinger, R., Nauhassenay, H., Berhane, Y., \& Keating, J. (2013). Travel history and malaria infection risk in a low-transmission setting in Ethiopia: a case control study. Malaria journal, 12(1), 33.

27. Nofal, S. D., Peto, T. J., Adhikari, B., Tripura, R., Callery, J., Bui, T. M., ... \& Pell, C. (2019). How can interventions that target forest-goers be tailored to accelerate malaria elimination in the Greater Mekong Subregion? A systematic review of the qualitative literature. Malaria journal, 18(1), 32. 
28. Rogawski, E. T., Congpuong, K., Sudathip, P., Satimai, W., Sug-aram, R., Aruncharus, S., ... \& Meshnick, S. R. (2012). Active case detection with pooled real-time PCR to eliminate malaria in Trat province, Thailand. The American journal of tropical medicine and hygiene, 86(5), 789-791.

29. Kheang, S. T., Lin, M. A., Lwin, S., Naing, Y. H., Yarzar, P., Kak, N., \& Price, T. (2018). Malaria case detection among mobile populations and migrant workers in Myanmar: comparison of 3 service delivery approaches. Global Health: Science and Practice, 6(2), 384-389.

30. Kyaw, A. M. M., Kathirvel, S., Das, M., Thapa, B., Linn, N. Y. Y., Maung, T. M., ... \& Thi, A. (2018). “AlertAudit-Act": assessment of surveillance and response strategy for malaria elimination in three low-endemic settings of Myanmar in 2016. Tropical medicine and health, 46(1), 11.

31. McLean, A. R., Wai, H. P., Thu, A. M., Khant, Z. S., Indrasuta, C., Ashley, E. A., ... \& Smithuis, F. M. (2018). Malaria elimination in remote communities requires integration of malaria control activities into general health care: an observational study and interrupted time series analysis in Myanmar. BMC medicine, 16(1), 110.

32. Von Seidlein, L., Peto, T. J., Landier, J., Nguyen, T. N., Tripura, R., Phommasone, K., ... \& Thwin, M. M. (2019). The impact of targeted malaria elimination with mass drug administrations on falciparum malaria in Southeast Asia: a cluster randomised trial. PLoS medicine, 16(2).

33. Landier, J., Kajeechiwa, L., Thwin, M. M., Parker, D. M., Chaumeau, V., Wiladphaingern, J., ... \& Cerqueira, D. (2017). Safety and effectiveness of mass drug administration to accelerate elimination of artemisininresistant falciparum malaria: a pilot trial in four villages of Eastern Myanmar. Wellcome open research, 2. 34. von Seidlein, L., Peto, T. J., Tripura, R., Pell, C., Yeung, S., Kindermans, J. M., ... \& Maude, R. (2019). Novel Approaches to Control Malaria in Forested Areas of Southeast Asia. Trends in parasitology.

35. Chen-Hussey, V., Carneiro, I., Keomanila, H., Gray, R., Bannavong, S., Phanalasy, S., \& Lindsay, S. W. (2013). Can topical insect repellents reduce malaria? A cluster-randomised controlled trial of the insect repellent $\mathrm{N}, \mathrm{N}$ diethyl-m-toluamide (DEET) in Lao PDR. PloS one, 8(8).

36. Crawshaw, A. F., Maung, T. M., Shafique, M., Sint, N., Nicholas, S., Li, M. S., ... \& Hii, J. (2017). Acceptability of insecticide-treated clothing for malaria prevention among migrant rubber tappers in Myanmar: a clusterrandomized non-inferiority crossover trial. Malaria journal, 16(1), 92.

37. Thang, N. D., Erhart, A., Speybroeck, N., Xa, N. X., Thanh, N. N., Van Ky, P., ... \& d'Alessandro, U. (2009). Long-lasting insecticidal hammocks for controlling forest malaria: a community-based trial in a rural area of central Vietnam. PLoS One, 4(10). 